International Journal of Pure and Applied Mathematics

Volume 85 No. 5 2013, 887-890

ISSN: $1311-8080$ (printed version); ISSN: 1314-3395 (on-line version)

url: http://www.ijpam.eu

doi: http://dx.doi.org/10.12732/ijpam.v85i5.7

ijpam.eu

\title{
ON THE MAXIMAL SYMMETRIC TENSOR RANK FOR MULTIVARIATE HOMOGENEOUS POLYNOMIALS
}

\author{
E. Ballico \\ Department of Mathematics \\ University of Trento \\ 38123 Povo (Trento) - Via Sommarive, 14, ITALY
}

\begin{abstract}
For all positive integers $m, d, b$ let $\rho(m, d)$ (resp. $\rho(m, d, b))$ be the maximal symmetric tensor rank of any $f \in \mathbb{C}\left[x_{0}, \ldots, x_{n}\right] \backslash\{0\}$ homogeneous of degree $d$ (resp. and with border rank $\leq b$ ). Here we prove that $\rho(m, d) \leq$ $\left(\begin{array}{c}m+d \\ m\end{array}\right)-m$ for all $m \geq 2$ and $d \geq 2$ (only by 1 better than a far more general result of Landsberg and Teitler), that $\rho(m, d, b) \leq \rho(b-1, d, b)$ if $2 \leq b \leq m$ and $d \geq b-1$ and that $\rho(m, d, b) \leq d \cdot\left\lceil\left(\begin{array}{c}m+d \\ m\end{array}\right) /(m+1)\right\rceil$ if $2 \leq b \leq m$ and $3 \leq d \leq b-2$.
\end{abstract}

AMS Subject Classification: 14N05, 14Q05, 15A69

Key Words: symmetric tensor rank, Veronese variety, secant variety, border rank

\section{Introduction}

Let $\mathbb{K}$ be an algebraically closed base field such that $\operatorname{char}(\mathbb{K})=0$. Let $Y \subset \mathbb{P}^{r}$ be an integral and non-degenerate subvariety. For each $P \in \mathbb{P}^{r}$ the $Y$-rank $r_{Y}(P)$ of $Y$. For all integers $m \geq 1, d>0$ let $\mathbb{K}\left[x_{0}, \ldots, x_{m}\right]_{d}$ denote the set of all homogeneous polynomials with degree $d$. This set is a $\mathbb{K}$-vector space with dimension $\left(\begin{array}{c}m+d \\ m\end{array}\right)$. We have $H^{0}\left(\mathbb{P}^{m}, \mathcal{O}_{\mathbb{P} m}(d)\right)=\mathbb{K}\left[x_{0}, \ldots, x_{m}\right]_{d}$. Hence

Received: March 5, 2013

(c) 2013 Academic Publications, Ltd. url: www.acadpubl.eu 
$\mathbb{K}\left[x_{0}, \ldots, x_{m}\right]_{d}$ induces the order $d$ Veronese embedding $\nu_{d}: \mathbb{P}^{m} \rightarrow \mathbb{P}^{\left(\begin{array}{c}m+d \\ m\end{array}\right)-1}$. Set $X_{m, d}:=\nu_{d}\left(\mathbb{P}^{m}\right)$ (the $m$-dimensional Veronese variety of order $d$ ). For any $f \in \mathbb{K}\left[x_{0}, \ldots, x_{m}\right]_{d} \backslash\{0\}$ the symmetric tensor rank $s r_{m, d}(f)$ of $f$ is the minimal integer $s>0$ such that $f=\sum_{i=1}^{s} \ell_{i}^{d}$ for some $\ell_{i} \in \mathbb{K}\left[x_{0}, \ldots, x_{m}\right]$. Obviously $s r_{m, d}(f)=s r_{m, d}(\lambda f)$ for all $\lambda \in \mathbb{K} \backslash\{0\}$. The non-zero polynomial $f$ corresponds to a unique $P \in \mathbb{P}^{\left(\begin{array}{c}m+d \\ m\end{array}\right)-1}$. We set $s r_{m, d}(P):=s r_{m, d}(f)$ and call it the symmetric tensor rank of $P$. Conversely, any $P \in \mathbb{P}_{\left(\begin{array}{c}m+d \\ m\end{array}\right)-1}$ corresponds to a unique set $\{\lambda g\}_{\lambda \in \mathbb{K} \backslash\{0\}}$ for some $g \in \mathbb{K}\left[x_{0}, \ldots, x_{m}\right]_{d} \backslash\{0\}$. Hence the symmetric tensor rank is defined for all points of $\mathbb{P}^{\left(\begin{array}{c}m+d \\ m\end{array}\right)-1}$. The definition of Veronese embedding gives $s r_{m, d}(P)=r_{X_{m, d}}(P)$ for all $P \in \mathbb{P}^{\left(\begin{array}{c}m+d \\ m\end{array}\right)-1}$. Let $\rho(m, d)$ be the maximal of all $s r_{m, d}(P), P \in \mathbb{P}^{\left(\begin{array}{c}m+d \\ m\end{array}\right)-1}$.

We first improve (but only by 1 ) the upper bound $\rho(m, d) \leq\left(\begin{array}{c}m+d \\ m\end{array}\right)-m+1$ for all positive integers $m, d([9]$, Corollary 5.2). Notice that this result by Landsberg and Teitler is just a very particular case of [9], Proposition 5.1.

Theorem 1. Fix integers $m \geq 2$ and $d \geq 2$. Then $\rho(m, d) \leq\left(\begin{array}{c}m+d \\ d\end{array}\right)-m$.

Remark 1. Take $m=d=2$ and let $Y:=X_{2,2} \subset \mathbb{P}^{5}$ be the Veronese surface. Theorem 1 says that $r_{Y}(P) \leq 3$ for all $P \in \mathbb{P}^{5}$. Since the secant variety of $X_{2,2}$ has dimension 4 , we have $r_{Y}(P) \geq 3$ for a general $P \in \mathbb{P}^{5}$. Hence $\rho(2,2)=3$.

Theorem 1 is just a particular case of the following result.

Proposition 1. Let $Y \subset \mathbb{P}^{r}$ be an integral and non-degenerate $m$ dimensional variety, $r-2 \geq m \geq 1$, such that $\operatorname{dim}(\operatorname{Sing}(Y)) \leq m-2$, with the convention $\operatorname{dim}(\emptyset)=-1$. Fix $P \in \mathbb{P}^{r}$ and set $E:=\left\{O \in Y_{\text {reg }}: P \in T_{O} Y\right\}$. Assume $\operatorname{dim}(E) \leq m-2$. Then $r_{Y}(P) \leq r-m$.

Now we add a constraint: the border rank. Fix any integral and nondegenerate variety $Y \subset \mathbb{P}^{r}$. For any integer $b>0$ the $b$-secant variety $\sigma_{b}(Y) \subseteq$ $\mathbb{P}^{r}$ is (by definition) the closure in $\mathbb{P}^{r}$ of of the union of all linear spaces $\langle S\rangle$, where $S$ is a subset of $Y$ with cardinality $b$. The algebraic set $\sigma_{b}(Y)$ is an integral variety and $\operatorname{dim}\left(\sigma_{b}(Y)\right) \leq \min \{r,(b+1) \cdot \operatorname{dim}(Y)-1\}$ for all $r, Y, b$. The dimensions of all varieties $\sigma_{b}\left(X_{m, d}\right)$ are known by a famous theorem of J. Alexander and A. Hirschowitz ([1], [2], [7]). The points $P \in \sigma_{b}\left(X_{m, d}\right) \backslash$ $\sigma_{m-1}\left(X_{m, d}\right)$ are said to have border rank $b$. Hence $\sigma_{b}\left(X_{m, d}\right)$ is the set of all $P \in \mathbb{P}^{\left(\begin{array}{c}m+d \\ m\end{array}\right)-1}$ with border rank $\leq b$. For all positive integers $m, d, b$ let $\rho(m, d, b)$ be the maximal integer $s r_{m, d}(P)$, where $P \in \sigma_{b}\left(X_{m, b}\right)$. Of course, $\rho(m, d, 1)=1$ for all $m, d, \rho(m, 1, b)=1$ for all $m$ and $b, \rho(m, d, b) \leq \rho(m, d)$ and and $\rho(m, d, b) \leq \rho(m, d, b+1)$ for all $m, d, b$. A theorem of Sylvester says 
that $\rho(1, d, b)=d$ for all $b \geq 2$ ([5], [8], [9], Theorem 4.1). In this note we prove the following result.

Theorem 2. Fix integers $m, d, b$ such that $2 \leq b \leq m$.

(i) If $d \geq b-1$, then $\rho(m, d, b) \leq \rho(m-1, d, b)$.

(ii) If $3 \leq d \leq b-2$, then $\rho(m, d, b) \leq d \cdot\left\lceil\left(\begin{array}{c}m+d \\ m\end{array}\right) /(m+1)\right\rceil$.

\section{The Proofs}

Proof of Proposition 1. If $P \in Y$, then $r_{Y}(P)=1$. Hence we may assume $P \notin Y$. The case $m=1$ is the main result of [3] (Notice that we use that $r \geq m+2=3$ in this case). Hence we may assume $m \geq 2$. Let $V \subset \mathbb{P}^{r}$ be a general linear subspace of codimension $m-1$ containing $P$. Since $P \notin Y$, the restriction to $Y$ of the linear system of all hyperplanes through $P$ has no base points. Hence Bertini's theorem implies $E \cap V=\emptyset, V \cap \operatorname{Sing}(Y)=\emptyset$ and that $C:=Y \cap V$ is an integral and smooth curve spanning $V$. Since $E \cap V=\emptyset, P$ is not contained in a tangent line of $C$. Hence $r_{C}(P) \leq \operatorname{dim}(V)-1=r-m$ ([3], Theorem 1) (again, we use that $r \geq m+2$ ). Since $r_{Y}(P) \leq r_{C}(P)$, we get $r_{Y}(P) \leq r-m$.

Proof of Theorem 1. Fix $P \in \mathbb{P}^{r}, r:=\left(\begin{array}{c}m+d \\ m\end{array}\right)-1$. The variety $X_{m, d} \subset \mathbb{P}^{r}$ is smooth and non-degenerate. Let $E:=\left\{O \in X_{m, d}: P \in T_{O} X_{m, d}\right\}$. By Proposition 1 Theorem 1 is true for $P$ if $\operatorname{dim}(E) \leq m-2$. In particular Theorem 1 is true for $P$ if $E=\emptyset$. If $E \neq \emptyset$, then $s r_{m, d}(P)=d$ by [5], Theorem 32 (the reader may check the proof even in the case $d=2$, i.e. in the case in which $E$ is the image of a line of $\left.\mathbb{P}^{m}\right)$. If $E=\emptyset$, then Proposition 1 gives $s r_{m, d}(P) \leq\left(\begin{array}{c}m+d \\ m\end{array}\right)-m$.

Proof of Theorem 2. First assume $d \geq b-1$. In this case there is a zerodimensional scheme $W \subset \mathbb{P}^{m}$ such that $\operatorname{deg}(W)=b$ and $P \in\left\langle\nu_{d}(W)\right\rangle$ ([5], Proposition 11, [6], Lemma 2.1.6). Set $M:=\langle W\rangle \subseteq \mathbb{P}^{m}$. Since $\operatorname{deg}(W)=b$, we have $a:=\operatorname{dim}(M) \leq b-1$. Since $P \in\left\langle\nu_{d}(W)\right\rangle$, we have $P \in\left\langle\nu_{d}(M)\right\rangle$. We have $s r_{m, d}(P)=r_{\nu_{d}(M)}(P)$ ([10], Proposition 3.1, for the non-symmetric case; see [8], Exercise 3.2.2.2, for a stronger statement). Hence $s r_{m, d}(P) \leq \rho(a, d)$. Notice that [8], Exercise 3.2.2.2, implies $\rho(a, d) \leq \rho(b-1, d)$. To conclude it is sufficient to prove that $P \in \sigma_{b}(M)$. By the proof of [5], Proposition 11, $W$ is smoothable inside $\mathbb{P}^{m}$. By [6], Proposition 2.1.5, $W$ is smoothable inside $M$. Hence $P \in \sigma_{b}(M)$ ([6], Lemma 2.1.6). 
Now assume $3 \leq d \leq b-2$. Since $d \geq 3$, we have $\rho(m, d) \leq d \cdot\left\lceil\left(\begin{array}{c}m+d \\ m\end{array}\right) /(m+\right.$ 1) $\rceil$ ([4], Theorem 1).

\section{Acknowledgements}

The author was partially supported by MIUR and GNSAGA of INdAM (Italy).

\section{References}

[1] J. Alexander, A. Hirschowitz, La méthode d'Horace éclatée: application à l'interpolation en degrée quatre, Invent. Math., 107 (1992), 585-602.

[2] J. Alexander, A. Hirschowitz, Polynomial interpolation in several variables, J. Alg. Geom., 4, No. 2 (1995), 201-222.

[3] E. Ballico, On the symmetric tensor rank of non-tangential points of projective curves, Int. J. Pure Appl. Math., 64, No. 2 (2010), 187-189.

[4] E. Ballico, An upper bound for the symmetric tensor rank of a low degree polynomial in a large number of variables, ISRN-Geometry, To Appear.

[5] A. Bernardi, A. Gimigliano, M. Idà, Computing symmetric rank for symmetric tensors, J. Symbolic. Comput., 46, No. 1 (2011), 34-53.

[6] J. Buczyński, A. Ginensky, J. M. Landsberg, Determinantal equations for secant varieties and the Eisenbud-Koh-Stillman conjecture, arXiv:1007.0192v4 [math.AG]; To appear in: J. London Math. Soc.

[7] K. Chandler, A brief proof of a maximal rank theorem for generic double points in projective space, Trans. Amer. Math. Soc., 353, No. 5 (2000), 1907-1920.

[8] J.M. Landsberg, Tensors: Geometry and Applications, Graduate Studies in Mathematics, Vol. 128, Amer. Math. Soc. Providence (2012).

[9] J.M. Landsberg, Z. Teitler, On the ranks and border ranks of symmetric tensors, Found. Comput. Math., 10, No. 3 (2010), 339-366.

[10] L.H. Lim, V. de Silva, Tensor rank and the ill-posedness of the best lowrank approximation problem, SIAM J. Matrix Anal. Appl., 30, No. 3 (2008), 1084-1127. 